\title{
Patient stress and satisfaction when allowed the presence of an accompanying person during endoscopy
}

\author{
Jesse Lachter ${ }^{1,2}$, Benjamin Ephraim Bluen ${ }^{1}$ \\ ${ }^{1}$ Bruce and Ruth Rappaport Faculty of Medicine, Technion-Israel Institute of Technology, Haifa, Israel \\ ${ }^{2}$ Gastroenterology Institute, Rambam Health Care Campus, Haifa, Israel \\ Email: J_Lachter@Rambam.health.Gov.IL, bbluen@gmail.com
}

Received 17 November 2012; revised 18 December 2012; accepted 24 December 2012

\begin{abstract}
Introduction: Patients undergoing esophagogastroduodenoscopy (EGD) have variable stress and anxiety and therefore commonly receive medications as sedative-anxiolytics. These medications have small but significant risks. To possibly make procedures safer and to increase patient satisfaction, this study aimed to measure the effects of allowing an accompanying person (AP) to be present in the endoscopy suite during EGD. Patients and Methods: Forty-two patients were randomly divided into two groups who would either be or not be offered to have an AP during endoscopy. Spielberger's well-validated state and trait anxiety evaluations were administered to patients before and after EGD. APs also completed questionnaires as to their reactions after EGD. Results: 84.2\% of the patients after EGD recommended accompaniment. Patients who underwent EGD with an AP tended $(p<0.06)$ to have decreased measurable anxiety compared to patients who were not offered an AP. The benefit was significant for persons with higher levels of anxiety prior to EGD $(p<0.04)$. Patients undergoing EGD for the first time had significantly more anxiety than those with previous EGD experience $(p<0.034)$. There was no significant reduction in sedative dosage when APs were present. Conclusions: Offering to permit the presence of an accompanying person during EGD was often shown to improve patient satisfaction and reduce anxiety.
\end{abstract}

Keywords: Endoscopy Escort; Sedation; Satisfaction

\section{INTRODUCTION}

It is understandable for patients to experience some stress preceding and during EGD. One appropriate way to reduce this anxiety is to use sedatives. Such medication has been found to have minimal but occasionally significant morbidity. However, based on a study in- cluding over 320,000 endoscopies, more than half of the major complications including mortality in endoscopic procedures may be attributable to the medications used [1].

Any intervention which may reduce anxiety, and thereby reduce the need for sedation, might therefore be hypothesized to help reduce morbidity and mortality. Anxiety, which is not easily quantified, has been made approximately measureable using physiologic parameters including heart rate, blood pressure and degree of sweating and by using validated questionnaires such as that of Spielberger, which test both state and trait anxiety [2]. High anxiety levels have various adverse effects not only in medical but also in daily situations, including negative affective response, more prolonged and difficult recoveries, and increased need for pain and anxiolytic medication [3]. Furthermore, reducing anxiety may reduce medical phobia (disproportionate fears) which might otherwise lead to avoidance of beneficial procedures [4]. This is especially true for patients undergoing their first endoscopic exam [5]. When patients feel lack of control, anxiety is increased. Reducing anxiety lessens the amount of time and support resources needed for recovery, which furthermore results in increased patient satisfaction and adherence to medical plans.

While the patient is irrefutably the primary client, a patient's surrounding environment includes significant others who may have a profound impact on his or her physical and mental health. The people who the patient knows and trusts may influence adherence to medical therapy. These "significant others" or APs may also be seen as secondary clients. Including these APs in part of any medical intervention such as EGD may help to achieve successful outcomes by leveraging their presence and role to help reduce the stress and anxiety of the patients undergoing endoscopy.

It is from the experience of the first author, having visited endoscopy centers worldwide that allowing the presence of an AP during EGD is quite rare in the United States, Canada, and in Western Europe with a notable 
exception to be in China where such practice is common. A questionnaire of 400 men and women ages 16 - 65 years old in England identified that the most important factor in the examination was the attitude of the doctor; allowing an AP during examinations was viewed by patients as a sign of respect [6]. Although the issues of anxiety and sedatives have been addressed at length by the American and European practice guidelines, they have not related to the influence of allowing an AP during EGD [7]. Therefore, two additional goals of this study are to raise awareness of this option and to encourage further investigation to assess the impact of patients' significant others in large-scale studies.

Attempts have been undertaken to reduce stress by various psychological preparations. Such preparations for EGD have included provision of information (orally and in pamphlets), modeling (seeing a videotape before EGD), hypnosis and other relaxation techniques [8]. As an unfortunate consequence of the modern healthcare system and training centers, over-worked house officers often either lack the time, the awareness, and/or the overall ability to optimally manage various patient social and behavioral concerns [9]. This further emphasizes the value of psychological preparations and the presence of a significant other during EGD as a low-cost method to reduce anxiety and perhaps thus also to reduce associated anesthesia-related morbidity.

The influence of psychological preparation for gastroscopy was investigated by Mahajam et al. in a study involving pediatric patients with a mean age of 12.2 years who underwent endoscopy. Studying a control group having regular preparation vs. additional psychological preparation including dolls of photography books simulating EGD procedures demonstrated statistically significant decreases in autonomic nervous system stimulation and procedural anxiety. This led to decreased parental anxiety and helped to strengthen doctor-patient relationships [10]. In a similar manner, the anxiety of a patient's significant other may be decreased by pre-procedural psychological preparation.

Individualization of approach and considering the holistic approach to a patient as a part of a social network that includes others is key to patient satisfaction. It is important to realize that patients vary in terms of the extent of worry over EGD. For example, younger patients have been shown to be most concerned about EGD-related pain and procedural complications whereas elderly patients were most concerned about potential findings [5]. Also key is to identify specific groups of patients who have a high risk of elevated anxiety levels prior to procedures. Increased anxiety is a phenomenon which has been shown to be particularly prevalent in certain patient groups, notably younger patients and those undergoing gastroenterological procedures for their first time. Extra emphasis should be placed on helping such individuals to relieve any excessive anxiety [6]. The outlined anxiety may be reduced by the presence of an AP may in turn allow the reduction or elimination of the use of anti-anxiety medications, most commonly benzodiazepines, which according to major studies are responsible for the majority of the morbidity and mortality from upper gastrointestinal endoscopy [1].

\section{AIM}

By having an accompanying person present in the endoscopy suite, persons who chose to have such an accompanying person present might become less anxious before and during the procedure. This study hypothesized that measureable anxiety and satisfaction of patients and APs within the medical encounter might be affected by offering this option to patients.

\section{PATIENTS AND METHODS}

Every patient was instructed to come for EGD with an accompanying person, without explanation for such being given. This is standard operating procedure, because anyone receiving sedation should be accompanied afterwards. 42 outpatients were randomized to either have offered to them the presence of an AP during the procedure or not. With the help of a trained interviewer, every patient prior to EGD underwent testing using the Spielberger anxiety scales for state anxiety and situational anxiety. The sedation used for these procedures was a standard dosage of fentanyl $0.05 \mathrm{mg}$ IV in addition to midazolam titrated until considered sufficiently effective (1 - $5 \mathrm{mg}$ ). No oral or topical spray anesthetic was used.

After EGD, the patients again completed a questionnaire measuring anxiety. Every patient had heart rate, blood, pressure, and degree of sweating noted once at the beginning and once at the end of the EGD. The accompanying persons also completed a questionnaire, including scaled questions regarding satisfaction with the medical encounter and evaluation of their experience. Data was analyzed using the Statistical Program for the Social Sciences (SPSS) and by $\mathrm{X}^{2}$ analysis.

This study was approved in advance by the Helsinki Committee (Institutional Review Board) of the Rambam Healthcare Campus. The study was not funded and thus there are no relevant disclosures.

\section{RESULTS}

Forty-two patients were selected with 23 controls and 19 subjects. The control group consisted of 56.5\% (13/22) male and $43.5 \%$ (10/23) female patients; the experimental group contained $47.4 \%$ male (9/19) and 52.6\% (10/19) female subjects. Patients were sampled from a variety of 
different ethnic backgrounds mostly including those from Israeli, Russian, Romanian, and Ethiopian heritages. APs consisted of mostly spouses (34\%), children (20\%), or family members (12\%).

After endoscopy, results from questionnaires filled out by patients who underwent EGD with the presence of an AP showed that $83 \%$ (16/19) of the experimental group patients recommended that procedural accompaniment be offered (see Table 1). Including the control group, a total of nearly $59 \%$ of subjects requested to have APs during EGD whereas 38\% declined, and 3\% of patients were undecided, which likely reflects the high anxiety rate in the pre-procedure waiting room and from EGD itself. After EGD, $72.2 \%$ of APs recommended having an AP present during EGD while $16.7 \%$ recommended against the presence of an AP during the procedure while 11.1\% were undecided (see Table 2).

Statistical analysis demonstrated that patients undergoing EGD for the first time had higher state anxiety than those who had previous endoscopy experience ( $\mathrm{p}<$ 0.034). This was accomplished by comparing the state anxiety $\mathrm{X}^{2}$ values. These were determined to be 2.86 for patients who underwent EGD for the first time and 2.16 for patients who had undergone prior EGD. Further analysis of state anxiety revealed that patients who underwent EGD with an accompanying person tended (trend $\mathrm{p}<$ 0.06 ) to have a lessening of state anxiety as compared to those who underwent EGD alone, with average state anxiety scores of controls being 2.30 vs. 2.42 of the experimental group. This also brought about a trend to increased patient satisfaction after the procedure, although this was not found to be statistically significant. The benefit of having an AP was greatest (and statistically significant $p<0.05$ ) for the subgroup of patients with the highest initial state anxiety. The overall patient and physician experience was summarized in the artwork in the accompanying Figures $\mathbf{1}$ and $\mathbf{2}$.

There were no statistically significant differences noted in the change of heart rate, sweating, or blood pressure between the two groups. Also, there was no reduction in the dose of any of the sedatives or analgesics used amongst the patients when allowed the presence of an AP.

\section{DISCUSSION}

Allowing the presence of an accompanying person during EGD came to be considered for research after many patients and their family members requested this spontaneously, and was based on the commonly observed obvious anxiety of many patients before EGD. After occasionally acceding to requests for the presence of an accompanying person, some endoscopists found that there was negligible if any interference in the procedure, although it should be noted that other endoscopists may feel uncomfortable with allowing the presence of an accompanying person during endoscopy.

Patients with high anxiety prior to EGD appeared to benefit from the presence of an AP during the procedure. $84 \%$ of experimental group patients and $72.2 \%$ of APs recommended for having accompaniment of a significant other during upper endoscopy (see Tables 1 and 2). The most profound benefit was seen in patients with an elevated pre-test anxiety who demonstrated a statistically significant decrease $(\mathrm{p}<0.05)$ in anxiety as compared to the same patient category without an AP. Although the optimal treatment for every patient's emotional status must be individualized, it appears that highly anxious patients benefit the most from an AP during EGD. Another group of patients that may benefit from an AP during EGD are those undergoing EGD for the first time. As might reasonably have been expected, this group displayed statistically significant $(\mathrm{p}<0.034)$ higher levels of anxiety than those who had previously underwent EGD. Patients and physicians alike expressed increased satisfaction from EGD likely due to the anxiety reduction most prominent in patients with high pre-procedural anxiety (see Figures 1 and 2).

In another high-anxiety setting in which the presence of APs was assessed was the emergency department. In evaluating AP satisfaction in the high-anxiety emergency department setting, $63.2 \%$ of APs to be very satisfied, 28.2\% slightly satisfied, and the remainder of APs being unsatisfied. It was also noted that these significant others also served to highlight the feeling of isolation of the patients in which they accompanied [11]. There, it was

Table 1. Did patients undergoing EGD recommend having a significant other present?

\begin{tabular}{|c|c|c|c|c|c|c|}
\hline Patient desire for escort & $\begin{array}{c}\text { Number of patients-experimental } \\
\text { group }\end{array}$ & Percent (\%) & $\begin{array}{l}\text { Number of patients-control } \\
\text { group }\end{array}$ & Percent (\%) & Total & Percent (\%) \\
\hline Yes & 16 & 84.2 & 4 & 26.7 & 20 & 58.8 \\
\hline No & 3 & 15.9 & 10 & 66.7 & 13 & 38.2 \\
\hline Undecided & 0 & 0 & 1 & 6.7 & 1 & 2.9 \\
\hline
\end{tabular}

Displayed in this chart are the recommendations of control and experimental group patients in regards to the presence of an AP during upper endoscopy. A key observation is that $84 \%$ of experimental group patients recommend having a significant other (AP) present during the procedure. 
Table 2. Recommendations of significant others after being present during EGD.

\begin{tabular}{ccc}
\hline For/against accompaniment & Frequency & Percent (\%) \\
\hline For & 13 & 72.2 \\
Against & 3 & 16.7 \\
Undecided & 2 & 11.1 \\
Total & 18 & 100.0 \\
\hline
\end{tabular}

Listed in the table are the recommendations of APs after EGD. Greater than $72 \%$ of APs advocated for accompaniment of a significant other during EGD.

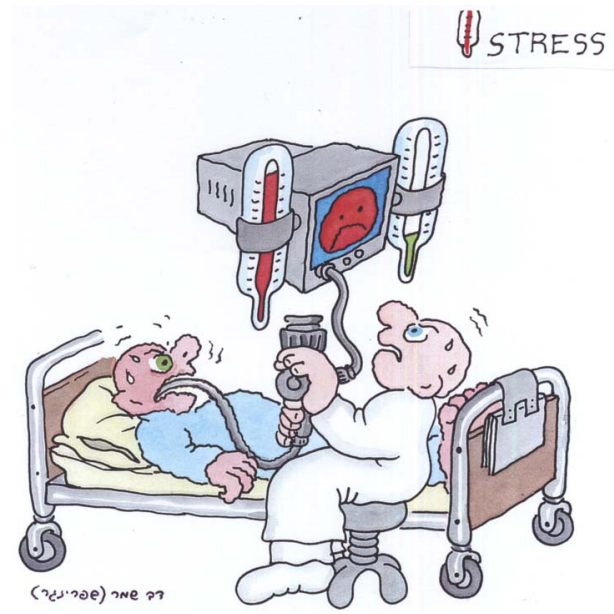

Figure 1. Illustrates how high-anxiety states of patients may affect EGD. Not only do the changes in heart rate and blood pressure affect the patient, but they also have deleterious consequences on the operating endoscopist that together may increase the overall complication rate.

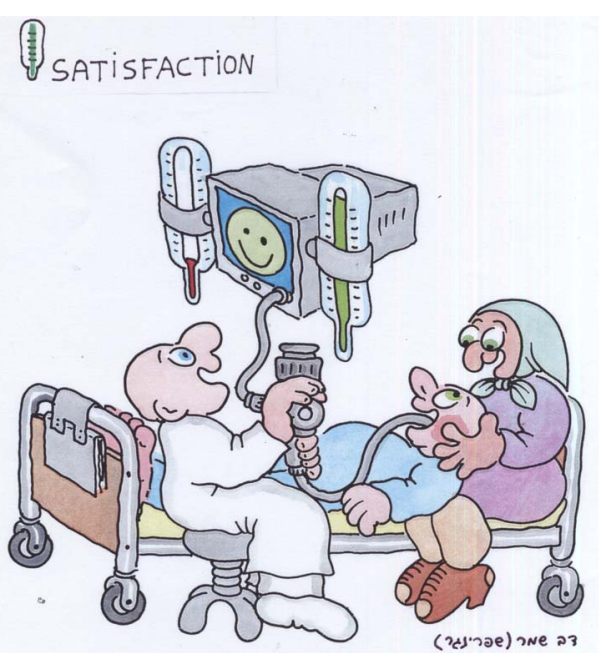

Figure 2. Conveys the potential benefits of having an AP present during EGD. Due to the APs influence, a now-calmer patient and a more comfortable performing endoscopist may result in reduced procedural and anesthesiarelated complications while increasing patient satisfaction. demonstrated that a lower anxiety level of APs correlated with increased satisfaction upon leaving the department [12].

The concept of having one's significant other present has been applied to obstetric wards as well. While personal preference may play a role, local experience showed that after several birthing centers allowed for accompanying persons during delivery, increased patient demand strongly encouraged allowing the presence of additional people in the delivery room amongst staff who otherwise would have preferred not to make such an allowance. This includes an AP present during forms of birth including assisted deliveries and Cesarean sections [13]. In regards to OB-GYN practice, it was demonstrated by Afaneh et al. that although a majority of patients (65\%) felt no need for an AP, a small but significant group of patients requested accompaniment regardless of the gender of the examiner. Therefore, patients should be offered the option of having an AP with their opinion respected and documented [14]. One can perceive additional settings and procedures including EGD that lead to high-anxiety states in which persons might come to feel entitled to have an accompanying person present.

In reducing patient stress and anxiety, it has been thought that this may lead to a reduction in the amount of anesthesia administered prior to and during EGD, thus improving procedural safety. As sedatives are more liberally administered in modern medicine, a much larger sample study population is needed to correlate respective sedative doses with their associated levels of anxiety. A potential alternative to this study would be to include a group of patients with an AP who would not receive any anesthesia which would then be compared to the other study groups. However, this would be a drastic change as compared to administering a half-dose of midazolam and then measuring whether this dose-reduction would lead to equivalent patient satisfaction and anxiety reduction. As the safety profile of the administered sedatives is quite good [15], power analysis suggests that a very large sample population is necessary to demonstrate potential benefits. This study was designed more of a "proof of concept" in that patients' significant others decrease anxiety instead of directly addressing the quantity of sedatives required to achieve equivalent results. Nevertheless, the presence of an AP decreasing patient anxiety and increasing procedural satisfaction are important areas of interest in gastroenterological procedures. In the emerging era of emphasis placed on quality indicators, such cost-effective improvements by a patient's significant other may be proven in larger follow-up studies to augment procedural efficiency and lessen EGD-related complications.

The present results were similar compared to a study by Shapiro and Tamir, who used non-validated qualita- 
tive comparisons of anxiety and fearfulness [16]. The present study utilized the Spielberger state and trait anxiety scores as quantitative indices, which have been validated in Hebrew and are widely used as benchmarks, were employed for a rigorous and generalizable assessment of anxiety [2]. In addition, the biological parameters of anxiety, heart rate blood pressure and sweating were also assessed. Both genders were evaluated equally as well as patients from diverse cultural backgrounds. As the reduction in anxiety particularly in high-anxiety patients was demonstrated to be similar in principle to the reduction in anxiety of having an AP present during a birthing procedure it may be offered to selected individuals as a low-cost therapy that may reduce overall anesthesia-related complications and strengthen patient adherence for future beneficial procedures.

The potential drawbacks of allowing an AP, such as taking phone calls or interfering with the procedure were not seen. It has been subsequent experience that only rarely must an endoscopist ask an AP to leave the room due to circumstances such as AP discomfort or AP deflecting of attention from the patient.

\section{LIMITATIONS}

This is a single center small sample size study, which involves one culture of people. Only a much larger study could be expected to show reduction of the major anesthesia-related side effects because they are uncommon. However, the culture is very diverse, and these numbers, while small already point to significant benefits which accrue to centers which allow the presence of an AP.

\section{CONCLUSIONS}

Offering to allow an accompanying person to be present during EGD tended to:

1) Reduce patient anxiety, especially for high-anxiety patients and first-timers.

2) Increase patient and family satisfaction with the endoscopy encounter.

\section{ACKNOWLEDGEMENTS}

The authors wish to acknowledge the contributions to this study of Dr. Hadas Wiseman, Psychologist at Haifa University, Israel, and to Dov Shperling Shemer, for the artwork which he drew and donated to accompany this article to tell its message in a pleasant, memorable, and lighthearted way.

\section{REFERENCES}

[1] Sharma, V.K., Nguyen, C.C., Crowell, M.D., Lieberman, D.A., de Garmo, P. and Fleisher, D.E. (2007) A national study of cardiopulmonary unplanned events after GI endoscopy. Gastrointestinal Endoscopy, 66, 27-34. doi:10.1016/j.gie.2006.12.040

[2] Spielberger, C.D., Gorssuch, R.L., Lushene, P.R., Vagg, P.R. and Jacobs, G.A. (1983) Manual for the state-trait anxiety inventory. Consulting Psychologists Press, Inc., Mountain View.

[3] Gattuso, S.M., Litt, M.D. and Fitagerald, T.E. (1992) Coping with gastrointestinal endoscopy: Self-efficacy enhancement and coping style. Journal of Clinical Psychology, 60, 133-139.

[4] Horne, D.J.L., Vatmanidis, P. and Careri, A. (1994) Preparing patients for invasive medical and surgical procedures 1: Adding behavioral and cognitive interventions. Behavioral Medicine, 20, 5-13. doi:10.1080/08964289.1994.9934610

[5] Shipley, R.H., Butt, J.H., Farbry, J.E. and Horwitz, B. (1977) Psychological preparation for endoscopy: Physiological and behavioral changes in patients with differing coping styles for stress. Gastrointestinal Endoscopy, 24, 9-13. doi:10.1016/S0016-5107(77)73427-3

[6] Whitford, D.L., Karim, M. and Thompson, G. (2001) Attitudes of patients toward the use of chaperones in primary care. British Journal of General Practice, 51, 381383.

[7] Ladas, S.D., Novis, B., Triantafyllou, K., Schoefl, R., Stanciu, C., Isaacs, P., Willich, S.N., Ronn, O., Dremel, H., Livadas, G., Egan, B.J., Boyacioglu, S., Selimovic, A., Pulanic, R., Karagiannis, J., Van Vooren, J.P., Kouroumalis, E., O’Morain, C., Nowak, A., Deviere, J., Malfertheiner, P. and Axon, A. (2007) Ethical issues in endoscopy: Patient satisfaction, safety in elderly patients, palliation, and relations with industry. Second European Symposium on Ethics in Gastroenterology and Digestive Endoscopy. Endoscopy, 39, 556-565.

[8] Berk, E.J. and Brandt, L.J. (2001) Patients attitudes and apprehensions about endoscopy: How to calm troubled waters. American Journal of Gastroenterology, 96, 280284. doi:10.1111/j.1572-0241.2001.03508.x

[9] Brody, D.S. (1980) Physician recognition of behavioral, psychological, and social aspects of medical care. Archives of Internal Medicine, 140, 1286-1289. doi:10.1001/archinte.140.10.1286

[10] Mahajan, L., Wyllie, R., Steffen, R., Kay, M., Kitacka, G., Dettore, J., Sarigot, S. and McCue, K. (1998) The effects of a psychological preparation program on anxiety in children and adolescents undergoing gastrointestinal endoscopy. Journal of Pediatric Gastroenterology \& Nutrition, 27, 161-165. doi:10.1097/00005176-199808000-00006

[11] Morales-Guijarro, A.M., Nogales-Cortes, M.D. and Perez Tirado, L. (2011) Satisfied companion, a quality indicator in emergencies. Revista de Calidad Asistencial, 26, 47-53. doi:10.1016/j.cali.2010.07.004

[12] Ekwall, A., Gerdtz, M. and Manias, E. (2009) Anxiety as a factor influencing satisfaction with emergency department care: Perspectives of accompanying persons. Journal of Clinical Nursing, 18, 3489-3497. doi:10.1111/j.1365-2702.2009.02873.x

[13] Keinan, G., Ezer, A. and Feigin, M. (1992) The Influence of situational and personal variables on the effectiveness 
of social support during childbirth. Anxiety Research, 4, 325-337. doi:10.1080/08917779208248800

[14] Afaneh, I., Sharma, V., McVey, R., Murphy, C. and Geary, M. (2010) The use of a chaperone in obstetrical and gynaecological practice. Iranian Red Crescent Medical Journal, 103, 137-139.

[15] Rex, D.K., Deenadayalu, V.P., Eid, E., Imperiale, T.F., Walker, J.A., Sandhu, K., Clarke, A.C., Hillman, L.C., Horiuchi, A., Cohen, L.B., Heuss, L.T., Peter, S., Beglinger, C., Sinnott, J.A., Welton, T., Rofail, M., Subei, I., Sleven, R., Jordan, P., Goff, J., Gertenberger, P.D., Mun- nings, H., Tagle, M., Sipe, B.W., Wehrmann, T., Di Palma, J.A., Occhipinti, K.E., Barbi, E., Riphaus, A., Amann, S.T., Tohda, G., McClellan, T., Thueson, C., Morse, J. and Meah, N. (2009) Endoscopist-directed administration of propofol: A worldwide safety experience. Gastroenterology, 137, 1229-1237. doi:10.1053/j.gastro.2009.06.042

[16] Shapira, M. and Tamir, A. (1996) Presence of family member during upper endoscopy: What do patients and escorts think? Journal of Clinical Gastroenterology, 22, 272-274. doi:10.1097/00004836-199606000-00006 